\title{
Testing nonlinear vacuum electrodynamics with Michelson interferometry
}

\author{
Gerold O. Schellstede* and Volker Perlick团 \\ ZARM, University of Bremen, Am Fallturm, 28359 Bremen, Germany \\ Claus Lämmerzah围 \\ ZARM, University of Bremen, Am Fallturm, 28359 Bremen, Germany and \\ Institute of Physics, University of Oldenburg, 26111 Oldenburg, Germany
}

\begin{abstract}
We discuss the theoretical foundations for testing nonlinear vacuum electrodynamics with Michelson interferometry. Apart from some nondegeneracy conditions to be imposed, our discussion applies to all nonlinear electrodynamical theories of the Plebański class, i.e., to all Lagrangians that depend only on the two Lorentz-invariant scalars quadratic in the field strength. The main idea of the experiment proposed here is to use the fact that, according to nonlinear electrodynamics, the phase velocity of light should depend on the strength and on the direction of an electromagnetic background field. There are two possible experimental setups for testing this prediction with Michelson interferometry. The first possibility is to apply a strong electromagnetic field to the beam in one arm of the interferometer and to compare the situation where the field is switched on with the situation where it is switched off. The second possibility is to place the whole interferometer in a strong electromagnetic field and to rotate it. If an electromagnetic field is placed in one arm, the interferometer could have the size of a gravitational wave detector, i.e., an arm length of several hundred meters. If the whole interferometer is placed in an electromagnetic field, one would have to do the experiment with a tabletop interferometer. As an alternative to a traditional Michelson interferometer, one could use a pair of optical resonators that are not bigger than a few centimeters. Then the whole apparatus would be placed in the background field and one would either compare the situation where the field is switched on with the situation where it is switched off or one would rotate the apparatus with the field kept switched on. We derive the theoretical foundations for these types of experiments, in the context of an unspecified nonlinear electrodynamics of the Plebański class, and we discuss their feasibility. A null result of the experiment would place bounds on the parameters of the theory. We specify the general results to some particular theories of the Plebański class; in particular, we give numerical estimates for Born, Born-Infeld and Heisenberg-Euler theories.
\end{abstract}

PACS numbers: 03.50.Kk,11.10.Lm

\section{INTRODUCTION}

Modifying an earlier idea by Born [1, in 1934 Born and Infeld 2] suggested a nonlinear modification of vacuum electrodynamics in order to get rid of the infinite self-energies of point particles that occur in the standard Maxwell theory. Their theory can be derived from a Lorentz-invariant Lagrangian. A few years later, Heisenberg and Euler [3] derived an effective Lagrangian, again Lorentz-invariant, from quantum electrodynamics. These are the two best known examples within the class of all Lorentz-invariant nonlinear electrodynamical theories. More generally, Plebański 4 and also Boillat 5 studied the whole class of nonlinear electrodynamical theories that can be derived from a Lagrangian depending only on the two Lorentz-invariant scalars that are quadratic in the field strength. This class is often referred to as Plebański nonlinear electrodynamics. For a review of the Born-Infeld theory we refer, e.g., to BiałynickiBirula [6].

\footnotetext{
* gerold.schellstede@zarm.uni-bremen.de Alternative Email: schellst@physik.fu-berlin.de

i volker.perlick@zarm.uni-bremen.de

$\ddagger$ claus.laemmerzahl@zarm.uni-bremen.de
}

The physical relevance of these nonlinear vacuum electrodynamical theories is being widely discussed in the literature. It is believed that at a certain field strength the Heisenberg-Euler deviations from standard Maxwell theory should be observable, and the Born-Infeld theory has gained increasing attention since it was realized by Tseytlin [7] that the Born-Infeld Lagrangian can be derived as an effective Lagrangian from some versions of string theory. Observable effects of (nonlinear) modifications of the vacuum Maxwell equations have been discussed for many years, at least since the Ph.D. thesis of Toll [8]. Up to now, the only effect predicted by such modified theories that has already been observed is light-by-light scattering (see [9]); further experiments are under way, e.g. with the Large Hadron Collider at CERN 10. There is also an ongoing experiment 11 aiming at verifying the birefringence in vacuo as predicted by the Heisenberg-Euler theory. Also, it might be possible to measure the influence of background fields on the propagation speed of light in the laboratory. For the case of the Born-Infeld theory, such experiments have been suggested with the help of wave guides by Ferraro 12 and with homogeneous magnetic background fields by Dereli and Tucker 13 .

In this paper we focus on another method for testing nonlinear electrodynamics and discuss its theoretical 
foundations in detail. The basic idea is to measure the influence of a (strong) background field on the propagation speed of light with the help of an interferometer. Such an experiment has been suggested and discussed already in five earlier papers 14 18. However, all of them restrict the theoretical discussion to the Heisenberg-Euler theory or, in the case of Denisov et al. [15, to the HeisenbergEuler and the Born-Infeld theory. What is still missing is a comprehensive derivation of the relevant equations that cover the whole Plebański class. The basic idea of the experiment is simple. In the standard Maxwell vacuum theory, which is linear, the superposition principle holds, so there is no influence of a background field on the propagation of light. In the nonlinear theories, however, the phase velocity of light depends on the strength of the background field and on the propagation direction relative to the background field. This can be tested with a Michelson interferometer: If a strong background field is switched on and off in one interferometer arm, or if the whole interferometer is being rotated in a strong background field, the interference pattern should change. A null result would place bounds on the possible deviations from standard Maxwell vacuum theory.

It is the purpose of this paper to develop the theoretical foundations for this experimental test for an unspecified nonlinear electrodynamical theory of the Plebański class. We will then specify to Born, Born-Infeld and Heisenberg-Euler theory.

Throughout this paper, we consider Minkowski space as the underlying space-time model. We work in inertial coordinates, so the Minkowski metric is $\left(\eta^{i k}\right)=$ $\operatorname{diag}(1,1,1,-1)$. We use Einstein's summation convention for Latin indices taking values 1,2,3,4 and for Greek indices taking values $1,2,3$. Indices are raised and lowered with the Minkowski metric. We will use Gaussian cgs units throughout, because they are most convenient for our theoretical investigations. In these units, $E, B, D$ and $H$ are all measured in the same units, $\sqrt{\mathrm{g}} /(\sqrt{\mathrm{cm}} \mathrm{s})$. The reader can easily convert the results into SI units with the help of the formulas $E=\sqrt{4 \pi \epsilon_{0}} E_{\mathrm{SI}}$, $B=\sqrt{4 \pi / \mu_{0}} B_{\mathrm{SI}}, D=\sqrt{4 \pi / \epsilon_{0}} D_{\mathrm{SI}}$ and $H=\sqrt{4 \pi \mu_{0}} H_{\mathrm{SI}}$ [19]. For example, for a field $X=10^{3} \sqrt{\mathrm{g}} /(\sqrt{\mathrm{cm} s})$ in Gaussian cgs units, where $X=E, B, D$, or $H$, one gets

$$
\begin{array}{cc}
E_{\mathrm{SI}}=3 \times 10^{7} \frac{\mathrm{V}}{\mathrm{m}}, & B_{\mathrm{SI}}=100 \mathrm{mT}, \\
D_{\mathrm{SI}}=3 \times 10^{-4} \frac{\mathrm{As}}{\mathrm{m}^{2}}, & H_{\mathrm{SI}}=8 \times 10^{4} \frac{\mathrm{A}}{\mathrm{m}} .
\end{array}
$$

The paper is organized as follows. In Sec. II we recall the basic equations for the propagation of light rays according to nonlinear electrodynamics. Then in Sec. III the suggested interferometer experiment is described and in Sec. IV some particular applications are discussed.

\section{LIGHT PROPAGATION IN NONLINEAR ELECTRODYNAMICS}

\section{A. The Plebański class of nonlinear electrodynamical theories}

The nonlinear electrodynamical theories which are at the center of our examination derive from an action

$$
S\left[A_{m}\right]=\frac{1}{4 \pi c} \int_{M}\left(\mathcal{L}\left(F_{m n}\right)+\frac{4 \pi}{c} j^{m} A_{m}\right) \mathrm{d} V_{4} .
$$

Here $j^{m}$ is a given current density, $A_{m}$ is the electromagnetic potential, $F_{m n}=\partial_{m} A_{n}-\partial_{n} A_{m}$ is the electromagnetic field strength and $\mathcal{L}$ is the Larangian for the electromagnetic field. Then the homogeneous group of Maxwell's equations is automatically satisfied,

$$
\partial_{[a} F_{b c]}=0
$$

Variation of the action with respect to the potential $A_{m}$ leads to the inhomogeneous group of Maxwell's equations,

$$
\partial_{b} H^{a b}=\frac{4 \pi}{c} j^{a}
$$

where

$$
H^{a b}=-\frac{\partial \mathcal{L}}{\partial F_{a b}}
$$

is the electromagnetic excitation. It is the constitutive law (5) that distinguishes different theories, while the Maxwell equations (3) and (4) are always the same.

Following Plebański [4, we require that the electromagnetic Lagrangian $\mathcal{L}$ depends on the electromagnetic field strength only via the Lorentz invariants

$$
F=\frac{1}{2} F_{m n} F^{m n} \quad \text { and } \quad G=-\frac{1}{4} F_{m n} \tilde{F}^{m n} .
$$

Here and in the following, the tilde denotes the Hodge dual,

$$
\tilde{F}^{m n}=\frac{1}{2} \varepsilon^{m n a b} F_{a b}
$$

As usual, $\varepsilon^{a b c d}$ is the totally antisymmetric Levi-Civita tensor with $\varepsilon^{1234}=-1$. Strictly speaking, only $F$ is invariant under all Lorentz transformations while $G$ changes sign under a parity transformation. Some authors restrict to Lagrangians that satisfy the equation $\mathcal{L}(F, G)=\mathcal{L}(F,-G)$ to assure invariance under parity transformations. However, for the purpose of this paper there is no need for this restriction.

The Plebański class contains, of course, the standard vacuum Maxwell theory which is given by the Lagrangian

$$
\mathcal{L}_{M}(F, G)=-\frac{1}{2} F
$$


As this theory is well tested for weak fields, many authors restrict their work to theories where the Lagrangian satisfies $\mathcal{L}+F / 2 \rightarrow 0$ for $F_{m n} \rightarrow 0$. Again, for our mathematical considerations there is no need for this restriction.

For a theory of the Plebański class the constitutive law (5) can be written, more specifically, as

$$
H^{a b}=-2 \mathcal{L}_{F} F^{a b}+\mathcal{L}_{G} \tilde{F}^{a b}
$$

where

$$
\mathcal{L}_{F}=\frac{\partial \mathcal{L}}{\partial F}, \quad \mathcal{L}_{G}=\frac{\partial \mathcal{L}}{\partial G} .
$$

Later we will also write

$$
\mathcal{L}_{F F}=\frac{\partial^{2} \mathcal{L}}{\partial F^{2}}, \quad \mathcal{L}_{G G}=\frac{\partial^{2} \mathcal{L}}{\partial G^{2}}, \quad \mathcal{L}_{F G}=\frac{\partial^{2} \mathcal{L}}{\partial F \partial G} .
$$

Additionally we will use a Hamiltonian formulation of the Plebański electrodynamics. For the special case of the Born-Infeld theory, the Hamiltonian formulation can be found in [2, 6] Whereas the Lagrangian depends on the field strength, the Hamiltonian depends on the excitation. Quite generally, for any theory based on a Lagrangian $\mathcal{L}\left(F_{m n}\right)$, the passage to the Hamiltonian formalism can be performed whenever the constitutive law (5) can be solved for $F_{m n}$. The Hamiltonian is then given by a covariant Legendre transformation,

$$
\mathcal{H}\left(H^{a b}\right)=-\frac{1}{2} H^{m n} F_{m n}-\mathcal{L}\left(F_{a b}\right)
$$

where, on the right-hand side, $F_{a b}$ has to be expressed in terms of $H^{m n}$ with the help of the constitutive law. For a theory of the Plebański class (i.e., if the Lagrangian depends only on $F$ and $G$ ), the Hamiltonian is a function of the two invariants

$$
R=-\frac{1}{2} H^{a b} H_{a b} \quad \text { and } \quad S=\frac{1}{4} H_{a b} \tilde{H}^{a b} .
$$

The relevant equations for the passage from the Lagrangian to the Hamiltonian description are given in the Appendix. There we will also give a criterion that guarantees that the constitutive law $(9$ can be solved for the field strength, at least locally.

\section{B. Three-dimensional notation of field equations}

In the following we will often use three-vector notation. The field strength has the three-dimensional representation

$$
E_{\alpha}=F_{\alpha 4}, \quad B^{\alpha}=\frac{1}{2} \varepsilon^{\alpha \beta \gamma} F_{\beta \gamma}=-\tilde{F}^{\alpha 4},
$$

where $\varepsilon^{\alpha \beta \gamma}$ is the totally antisymmetric spatial LeviCivita tensor, $\varepsilon^{123}=1$. With $E^{2}=\delta^{\mu \nu} E_{\mu} E_{\nu}, B^{2}=$ $\delta_{\mu \nu} B^{\mu} B^{\nu}$ and $\mathbf{E} \cdot \mathbf{B}=E_{\mu} B^{\mu}$, the invariants 6 read

$$
F=B^{2}-E^{2}, \quad G=\mathbf{B} \cdot \mathbf{E} .
$$

Analogously we write for the excitation

$$
D^{\alpha}=-H^{\alpha 4}, \quad H_{\alpha}=\frac{1}{2} \varepsilon_{\alpha \beta \gamma} H^{\beta \gamma}=\tilde{H}_{\alpha 4},
$$

which implies that the invariants 13 are given by

$$
R=D^{2}-H^{2}, \quad S=\mathbf{D} \cdot \mathbf{H}
$$

Then the constitutive law $(9)$ reads

$$
\begin{aligned}
& D_{\alpha}=-2 \mathcal{L}_{F} E_{\alpha}+\mathcal{L}_{G} B_{\alpha}, \\
& H_{\alpha}=-2 \mathcal{L}_{F} B_{\alpha}-\mathcal{L}_{G} E_{\alpha} .
\end{aligned}
$$

\section{Phase velocity and characteristic differential equation for $\mathcal{L}(\mathbf{F}, \mathbf{G})$ theories}

The characteristic surfaces determined by a set of partial differential equations can be defined as the hypersurfaces along which the solutions may have discontinuities. As an alternative, the characteristic surfaces can also be defined with the help of approximate plane waves; in this second approach, they come about as the high-frequency limit of the surfaces of constant phase. In view of applications to electrodynamics, the first approach is discussed, e.g., in the book by Hehl and Obukhov [20. The characteristic surfaces are hypersurfaces $\psi=$ constant, where the gradient of $\psi$ has to satisfy, at each point of space-time, a fourth-order equation which is known as the dispersion relation or as the Fresnel equation. If viewed as a partial differential equation for $\psi$, this equation is usually called the characteristic equation or the eikonal equation. Using this approach, Obukhov and Rubilar [21] have determined the Fresnel equation (i.e., the characteristic equation) for an arbitrary $\mathcal{L}(F, G)$ theory. Earlier, Novello et al. 22] had found an equivalent result in a different way. Their results show that, with the exception of a few special cases, theories of the Plebański class predict birefringence in vacuo. For background material on birefringence, and bimetricity, we refer to Visser et al. 23] and, for the particular case of the Heisenberg-Euler theory, to Dittrich and Gies [24] and to Shore [25].

Here we want to briefly sketch how the Fresnel equation of an arbitray $\mathcal{L}(F, G)$ theory can be derived with the help of an approximate-plane-wave ansatz. This is methodically different from the work of Obukhov and Rubilar 21] and Novello et al. 22] but it leads to the same result. The general method goes back to Luneburg and is outlined, e.g., for electrodynamics in ordinary media, in the book by Kline and Kay [26]. For a discussion in a more general context, which includes the case to be considered here, we refer to Perlick [27].

We consider a one-parameter family of electromagnetic fields of the form

$$
\begin{aligned}
F^{\prime} a b & \left(x^{m}\right)=F^{a b}\left(x^{m}\right) \\
+\operatorname{Re} & \left\{e^{-i \psi\left(x^{m}\right) / \lambda} \sum_{N=1}^{\infty}\left(\lambda^{N} F_{N}^{a b}\left(x^{m}\right)\right)\right\},
\end{aligned}
$$


where $F^{a b}$ is a given background field. $\lambda$ is a real bookkeeping parameter that is introduced in a way such that the high-frequency limit corresponds to $\lambda \rightarrow 0$. The summation sign in (19) is to be understood in the sense of an asymptotic series and not in the sense of a convergent series. While the amplitudes $F_{N}^{a b}$ are in general complex, the eikonal function $\psi$ is real. It gives the surfaces of constant phase, $\psi\left(x^{m}\right)=\psi\left(x^{\mu}, t\right)=$ constant. In 3-space, the normal to these surfaces is

$$
n_{\alpha}=\frac{\partial_{\alpha} \psi}{\sqrt{\left(\partial_{\beta} \psi\right)\left(\partial^{\beta} \psi\right)}} .
$$

The phase velocity $v_{\mathrm{P}}^{\alpha}$ can be introduced as the 3 -vector that gives the traveling speed of such a surface in the direction of its normal,

$$
v_{\mathrm{P}}^{\alpha}=-\frac{\partial^{\alpha} \psi}{\left(\partial_{\beta} \psi\right)\left(\partial^{\beta} \psi\right)} \frac{\partial \psi}{\partial t} .
$$

Feeding the ansatz 19 into Maxwell's equations and comparing equal powers of $\lambda$ gives a hierarchy of equations. In the lowest nontrivial order, which is known as the geometric optics approximation, one gets a first-order partial differential equation for $\psi$ which is the desired characteristic equation.

If this program is carried through for an $\mathcal{L}(F, G)$ theory, one finds the following result which is in agreement with Obukhov and Rubilar's [21]. The characteristic equation reads

$$
\begin{aligned}
\mathcal{L}_{F}\{M & \eta^{i j} \eta^{k l}+N \eta^{i j} F^{k m} F_{m}^{l} \\
& \left.+P F^{i m} F_{m}^{j} F^{k n} F_{n}^{l}\right\} p_{i} p_{j} p_{k} p_{l}=0
\end{aligned}
$$

where $p_{i}=\partial_{i} \psi$ and

$$
\begin{gathered}
M=\mathcal{L}_{F}^{2}+2 \mathcal{L}_{F} \mathcal{L}_{F G} G-\frac{1}{2} \mathcal{L}_{F} \mathcal{L}_{G G} F \\
+\left(\mathcal{L}_{F G}^{2}-\mathcal{L}_{F F} \mathcal{L}_{G G}\right) G^{2} \\
N=2 \mathcal{L}_{F} \mathcal{L}_{F F}+\frac{1}{2} \mathcal{L}_{F} \mathcal{L}_{G G} \\
\quad+\left(\mathcal{L}_{F G}^{2}-\mathcal{L}_{F F} \mathcal{L}_{G G}\right) F \\
P=\mathcal{L}_{F F} \mathcal{L}_{G G}-\mathcal{L}_{F G}^{2} .
\end{gathered}
$$

If $M$ has no zeros, 22 can be factorized as

$$
\mathcal{L}_{F} M\left(a_{1}^{i j} p_{i} p_{j}\right)\left(a_{2}^{k \ell} p_{k} p_{\ell}\right)=0
$$

where

$$
a_{A}^{i k}=\eta^{i k}+\sigma_{A} F^{i m} F_{m}^{k}
$$

for $A=1,2$ and

$$
\sigma_{1 / 2}=\frac{N}{2 M} \pm \sqrt{\frac{N^{2}}{4 M^{2}}-\frac{P}{M}} .
$$

In the following we restrict ourselves to Lagrangians such that $M$ and $\mathcal{L}_{F}$ have no zeros. This excludes some degenerate cases which are hardly of physical interest. Then the characteristic equation is equivalent to (which is up to conformal transformations in agreement with the results of Novello et al. [22])

$$
a_{A}^{i k} p_{i} p_{k}=0, \quad A=1,2
$$

and is sometimes called the "light-cone condition" (compare for example [24]). Generalizing a standard terminology from electrodynamics in media, $a_{1}^{i k}$ and $a_{2}^{i k}$ are called the optical metrics of the vacuum in the $\mathcal{L}(F, G)$ theory. If the two optical metrics do not coincide, i.e., if $\sigma_{1} \neq \sigma_{2}$, there is birefringence in vacuum. If one considers the next order in the above-mentioned hierarchy of equations, one sees that the case $A=1$ and the case $A=2$ correspond to two different polarization directions. Note that $\sigma_{1}$ and $\sigma_{2}$ are always real, because

$$
\begin{gathered}
N^{2}-4 M P= \\
\left(2 \mathcal{L}_{F} \mathcal{L}_{F F}-\frac{1}{2} \mathcal{L}_{F} \mathcal{L}_{G G}-P F\right)^{2}+4\left(\mathcal{L}_{F} \mathcal{L}_{F G}-P G\right)^{2}
\end{gathered}
$$

is a sum of two squares, and that $\sigma_{1}$ and $\sigma_{2}$ depend only on the two field invariants $F$ and $G$. In the standard vacuum Maxwell theory we have $\sigma_{1}=\sigma_{2}=0$, so these two functions characterize the deviation of our $\mathcal{L}(F, G)$ theory from the standard theory at the level of geometric optics.

If the Lagrangian is of the special form $\mathcal{L}(F, G)=$ $\mathcal{L}(\alpha F+\beta G)$ with some constant factors $\alpha$ and $\beta$, one has $P=0$ and therefore $\sigma_{1}=0$, i.e., one polarization mode behaves as in the standard Maxwell vacuum theory. This is true, in particular, if the Lagrangian is independent of $G$. (It is also true if the Lagrangian is independent of $F$ but this case was excluded by our assumption $\mathcal{L}_{F} \neq 0$.) Also, it is interesting to remark that two Lagrangians $\mathcal{L}$ and $\mathcal{L}+\beta G$ give the same characteristic equation, i.e. the two cases are not distinguishable at the level of geometrical optics. Of course, if one restricts to parity invariant Lagrangians adding a term of the form $\beta G$ is forbidden.

For some of our applications it will be desirable to write the optical metrics in terms of the excitation, rather than in terms of the field strength. It is then recommendable to start from a Hamiltonian formulation. It was mentioned already at the end of Sec. II A that the Plebański class of theories can be written in terms of a Hamiltonian $\mathcal{H}(R, S)$ rather than in terms of a Lagrangian $\mathcal{L}(F, G)$. In the Appendix we derive some replacement rules of how the relevant Hamiltonian expressions can be found from the Lagrangian expressions. By applying these replacement rules, we find that the optical metrics can be rewritten as

$$
a_{A}^{i k}=\eta^{i k}+\hat{\sigma}_{A} \tilde{H}^{i m} \tilde{H}_{m}^{k}
$$

where

$$
\hat{\sigma}_{A}=\frac{\hat{N}}{2 \hat{M}} \pm \sqrt{\frac{\hat{N}^{2}}{4 \hat{M}^{2}}-\frac{\hat{P}}{\hat{M}}}
$$


with the abbreviations

$$
\begin{gathered}
\hat{M}=\mathcal{H}_{R}^{2}+2 \mathcal{H}_{R} \mathcal{H}_{R S} S-\frac{1}{2} \mathcal{H}_{R} \mathcal{H}_{S S} R \\
+\left(\mathcal{H}_{R S}^{2}-\mathcal{H}_{R R} \mathcal{H}_{S S}\right) S^{2} \\
\hat{N}=2 \mathcal{H}_{R} \mathcal{H}_{R R}+\frac{1}{2} \mathcal{H}_{R} \mathcal{H}_{S S} \\
+\left(\mathcal{H}_{R S}^{2}-\mathcal{H}_{R R} \mathcal{H}_{S S}\right) R \\
\hat{P}=\mathcal{H}_{R R} \mathcal{H}_{S S}-\mathcal{H}_{R S}^{2} .
\end{gathered}
$$

In the following it will be convenient to use the threevector notation of (14) and (16). We decompose each of the 3-vectors $\boldsymbol{E}, \boldsymbol{B}, \boldsymbol{D}$, and $\boldsymbol{H}$ into amplitude and direction,

$$
\begin{aligned}
& \boldsymbol{B}\left(x^{\mu}, t\right)=B\left(x^{\mu}, t\right) \boldsymbol{v}\left(x^{\mu}, t\right), \\
& \boldsymbol{E}\left(x^{\mu}, t\right)=E\left(x^{\mu}, t\right) \boldsymbol{w}\left(x^{\mu}, t\right), \\
& \boldsymbol{H}\left(x^{\mu}, t\right)=H\left(x^{\mu}, t\right) \boldsymbol{r}\left(x^{\mu}, t\right), \\
& \boldsymbol{D}\left(x^{\mu}, t\right)=D\left(x^{\mu}, t\right) \boldsymbol{s}\left(x^{\mu}, t\right),
\end{aligned}
$$

where $|\boldsymbol{v}|=|\boldsymbol{w}|=|\boldsymbol{r}|=|\boldsymbol{s}|=1$. The spatial and temporal parts of $F^{i m} F_{m}^{k} p_{i} p_{k}$, which enter into 24, can then be written as

$$
\begin{gathered}
F^{\alpha m} F_{m}^{\beta} p_{\alpha} p_{\beta}=B^{2}\left[\boldsymbol{p} \cdot \boldsymbol{p}-(\boldsymbol{v} \cdot \boldsymbol{p})^{2}\right]-E^{2}(\boldsymbol{w} \cdot \boldsymbol{p})^{2} \\
F^{4 m} F^{\beta}{ }_{m} p_{\beta}=B E \boldsymbol{p} \cdot(\boldsymbol{w} \times \boldsymbol{v}) \\
F^{4 m} F_{m}^{4}=E^{2}
\end{gathered}
$$

Similarly,

$$
\begin{gathered}
\tilde{H}^{\alpha m} \tilde{H}_{m}^{\beta} p_{\alpha} p_{\beta}=D^{2}\left[\boldsymbol{p} \cdot \boldsymbol{p}-(\boldsymbol{s} \cdot \boldsymbol{p})^{2}\right]-H^{2}(\boldsymbol{r} \cdot \boldsymbol{p})^{2} \\
\tilde{H}^{4 m} \tilde{H}^{\beta}{ }_{m} p_{\beta}=D H \boldsymbol{p} \cdot(\boldsymbol{s} \times \boldsymbol{r}) \\
\tilde{H}^{4 m} \tilde{H}_{m}^{4}=H^{2}
\end{gathered}
$$

which will be used later.

\section{Rayvelocity and Hamilton equations for the rays}

Interpreting $Q_{A}=a_{A}^{i k} p_{i} p_{k}$ as a Hamiltonian, the characteristic partial differential equation $a_{A}^{i k} \partial_{i} \psi \partial_{k} \psi=0$ can be viewed as a Hamilton-Jacobi equation. The corresponding set of Hamilton equations, or canonical equations, determines the bicharacteristic curves or rays. For background material on the notions of characteristics and bicharacteristics we refer to Courant and Hilbert [28].

The rays are defined with respect to $Q_{1}$ and $Q_{2}$ separately, i.e., they depend on the polarization. The canonical equations read

$$
\frac{\mathrm{d} x^{a}}{\mathrm{~d} s}=\frac{\partial Q_{A}}{\partial p_{a}}, \quad \frac{\mathrm{d} p_{a}}{\mathrm{~d} s}=-\frac{\partial Q_{A}}{\partial x^{a}} .
$$

Here $s$ is a parameter along the rays which has no obvious physical meaning. In the following it will be convenient to reparametrize the rays by the time coordinate $t$, cf. 28. In order to do this, we have to assume that the rays of the Hamiltonian $Q_{A}$ are causal (i.e., timelike or lightlike) with respect to the Minkowski background metric. It was shown by Obukhov and Rubilar 21 that the optical metrics are always of Lorentzian signature, provided that we exclude the pathological cases where they degenerate. However, no convenient criterion on the Lagrangian $\mathcal{L}(F, G)$ seems to be known that guarantees causality of the rays with respect to the background metric. We will investigate this question in a separate paper; here we just restrict our discussion, from now on, to Lagrangians where the rays of the optical metrics are causal with respect to the background Minkowski metric.

Then it is guaranteed that $a_{A}^{44}<0$ and we may write the optical metrics as

$$
a_{A}^{i k} p_{i} p_{k}=\frac{a_{A}^{44}}{c^{2}}\left(c p_{4}+H_{A}^{+}\right)\left(c p_{4}+H_{A}^{-}\right)
$$

where

$$
H_{A}^{ \pm}=c\left(\frac{a_{A}^{\alpha 4} p_{\alpha}}{a_{A}^{44}} \pm \sqrt{\left(\frac{a_{A}^{\alpha 4} p_{\alpha}}{a_{A}^{44}}\right)^{2}-\frac{a_{A}^{\alpha \beta} p_{\alpha} p_{\beta}}{a_{A}^{44}}}\right)
$$

Equation 36 corresponds to splitting the null cone of the optical metric $a_{A}^{i k}$ into a future and a past cone. If we restrict our work here to future-oriented rays, we can write the characteristic equation as

$$
c p_{4}+H_{A}^{+}=0
$$

and the canonical equations read

$$
\frac{\mathrm{d} x^{\alpha}}{\mathrm{d} t}=\frac{\partial H_{A}^{+}}{\partial p_{\alpha}}, \quad \frac{\mathrm{d} p_{\alpha}}{\mathrm{d} t}=-\frac{\partial H_{A}^{+}}{\partial x^{\alpha}},
$$

$$
\frac{\mathrm{d} x^{4}}{\mathrm{~d} t}=c, \quad \frac{\mathrm{d} p_{4}}{\mathrm{~d} t}=-\frac{\partial H_{A}^{+}}{\partial x^{4}}
$$

If $a_{A}^{i k}$ is known, integration of 39 gives the spatial paths of the rays. The first equation of (40) says that the new parameter $t$ coincides with the coordinate time, while the second equation gives the change of the frequency of light.

The ray velocity can be read from (39),

$$
v_{\mathrm{S}}^{\alpha}:=\frac{\mathrm{d} x^{\alpha}}{\mathrm{d} t}=\frac{\partial H_{A}^{+}}{\partial p_{\alpha}} .
$$

The phase velocity 21 can be rewritten in terms of the Hamiltonian as

$$
v_{\mathrm{P}}^{\beta}=-\frac{c p_{4}}{p_{\alpha} p^{\alpha}} p^{\beta}=\frac{H_{A}^{+}}{p_{\alpha} p^{\alpha}} p^{\beta} .
$$


Phase and ray velocity coincide if and only if

$$
\frac{H_{A}^{+}}{p_{\alpha} p^{\alpha}} p^{\beta}=\frac{\partial H_{A}^{+}}{\partial p_{\beta}}
$$

which is true if and only if $H_{A}^{+}$is of the form

$$
H_{A}^{+}=f\left(x^{\mu}, c t\right) \sqrt{p_{\alpha} p^{\alpha}}
$$

where $f$ is any function of the space-time coordinates. Equation (44) is satisfied in the usual vacuum theory of Maxwell but not in general in other $\mathcal{L}(F, G)$ theories. Note that 44) implies

$$
\frac{\mathrm{d} x^{\alpha}}{\mathrm{d} t}=\frac{\partial H_{A}^{+}}{\partial p_{\alpha}}=\frac{f\left(x^{\mu}, c t\right) p^{\alpha}}{\sqrt{p^{\beta} p_{\beta}}},
$$

i.e., the condition $v_{\mathrm{S}}^{\alpha}=v_{\mathrm{P}}^{\alpha}$ can hold only if $d x^{\alpha} / d t$ and $p^{\alpha}$ are parallel.

\section{E. Parallel electric and magnetic fields}

We consider now the special case that $\boldsymbol{E}$ and $\boldsymbol{B}$ are parallel, i.e., that $\boldsymbol{v}=\boldsymbol{w}$ in the notation of (32). This case covers, of course, in particular the situation that one of the two field strengths, $\boldsymbol{E}$ or $\boldsymbol{B}$, is zero. With the aid of the transformation 138, described in the Appendix, we will then discuss, at the end of this section, the case that the excitations $\boldsymbol{D}$ and $\boldsymbol{H}$ are parallel.

If we specialize 33 to the case $\boldsymbol{v}=\boldsymbol{w}$ and insert the result into (25), the optical metrics read

$$
\begin{aligned}
a_{A}^{i k} p_{i} p_{k}=-\left(1-\sigma_{A} E^{2}\right) p_{4}^{2} & +\left(1+\sigma_{A} B^{2}\right) \boldsymbol{p}^{2} \\
& -\sigma_{A}\left(B^{2}+E^{2}\right)(\boldsymbol{w} \cdot \boldsymbol{p})^{2} .
\end{aligned}
$$

Hence, the Hamiltonian $H_{A}^{+}$from $(37)$ simplifies to

$$
H_{A}^{+}=c \sqrt{\frac{\left(1+\sigma_{A} B^{2}\right)}{\left(1-\sigma_{A} E^{2}\right)}\left(|\boldsymbol{p}|^{2}-(\boldsymbol{w} \cdot \boldsymbol{p})^{2}\right)+(\boldsymbol{w} \cdot \boldsymbol{p})^{2}}
$$

and the phase velocity 42 reads

$$
v_{\mathrm{P}}=c \sqrt{\frac{\left(1+\sigma_{A} B^{2}\right)}{\left(1-\sigma_{A} E^{2}\right)}\left(1-\frac{(\boldsymbol{w} \cdot \boldsymbol{p})^{2}}{|\boldsymbol{p}|^{2}}\right)+\frac{(\boldsymbol{w} \cdot \boldsymbol{p})^{2}}{|\boldsymbol{p}|^{2}}} .
$$

If we assume, in addition, that the unit vector of the background field is homogeneous, $\partial_{\alpha} w^{\beta}=0$, and that the amplitudes of the field strengths change only in the direction of $\boldsymbol{p}, \operatorname{grad} B \propto \boldsymbol{p}$ as well as $\operatorname{grad} E \propto \boldsymbol{p}$, the canonical equations 39 reduce to

$$
\begin{aligned}
\frac{\mathrm{d} x^{\alpha}}{\mathrm{d} t}=\frac{c}{H_{A}^{+}}\left\{\frac{\left(1+\sigma_{A} B^{2}\right)}{\left(1-\sigma_{A} E^{2}\right)}\left(p^{\alpha}-(\boldsymbol{w} \cdot \boldsymbol{p}) w^{\alpha}\right)\right. \\
\left.\quad+(\boldsymbol{w} \cdot \boldsymbol{p}) w^{\alpha}\right\} ; \quad \frac{\mathrm{d} p_{\alpha}}{\mathrm{d} t} \propto p_{\alpha} .
\end{aligned}
$$

The last equation implies that the direction of $p_{\alpha}$ is preserved along the ray.
If additionally the background fields are static, $\partial \boldsymbol{E} / \partial t=\mathbf{0}$ and $\partial \boldsymbol{B} / \partial t=\mathbf{0}$, the second equation of 40 reduces to

$$
\frac{\mathrm{d} p_{4}}{\mathrm{~d} t}=0
$$

which means that, in this case, the background fields do not change the frequency of light.

We are now interested in the special case that (44) holds which guarantees that phase velocity and ray velocity are equal and that $d x^{\alpha} / d t$ is parallel to $p^{\alpha}$. As the direction of $p^{\alpha}$ is preserved, the ray must then be a straight line.

There are two main cases where the Hamiltonian takes the form of (44). First, if $\boldsymbol{p} \| \boldsymbol{w}$, we find from (47), 48) and 49 that $H_{A}^{+}=c|\boldsymbol{p}|, v_{\mathrm{P}}=c$, and $\mathrm{d} x^{\alpha} / \mathrm{d} t=c p^{\alpha}|| \boldsymbol{p} \mid$, i.e., in this case the background fields have no effect. Second, if $\boldsymbol{p} \cdot \boldsymbol{w}=0$, one gets

$$
\begin{aligned}
& H_{A}^{+}=c|\boldsymbol{p}| \sqrt{\frac{1+\sigma_{A} B^{2}}{1-\sigma_{A} E^{2}}}, \\
& v_{\mathrm{P}}=c \sqrt{\frac{1+\sigma_{A} B^{2}}{1-\sigma_{A} E^{2}}}, \\
& \frac{\mathrm{d} x^{\alpha}}{\mathrm{d} t}=c \sqrt{\frac{1+\sigma_{A} B^{2}}{1-\sigma_{A} E^{2}} \frac{p^{\alpha}}{|\boldsymbol{p}|}} .
\end{aligned}
$$

This is the case which is most appropriate for the proposed experiment, because in this case one achieves two goals: the rays do not deviate from a straight line but the phase velocity does change in comparison to the Maxwell standard vacuum theory.

Now we go over to the case that $\boldsymbol{D}$ and $\boldsymbol{H}$ are parallel, i.e.., that $\boldsymbol{r}=\boldsymbol{s}$. With the help of 138 from the Appendix we find that in this case 47) and (48) have to be replaced with

$$
\begin{aligned}
H_{A}^{+} & =c \sqrt{\frac{\left(1+\hat{\sigma}_{A} D^{2}\right)}{\left(1-\hat{\sigma}_{A} H^{2}\right)}\left(|\boldsymbol{p}|^{2}-(\boldsymbol{s} \cdot \boldsymbol{p})^{2}\right)+(\boldsymbol{s} \cdot \boldsymbol{p})^{2}} \\
v_{\mathrm{P}} & =c \sqrt{\frac{\left(1+\hat{\sigma}_{A} D^{2}\right)}{\left(1-\hat{\sigma}_{A} H^{2}\right)}\left(1-\frac{(\boldsymbol{s} \cdot \boldsymbol{p})^{2}}{|\boldsymbol{p}|^{2}}\right)+\frac{(\boldsymbol{s} \cdot \boldsymbol{p})^{2}}{|\boldsymbol{p}|^{2}}}
\end{aligned}
$$

As above one gets for homogeneous and timeindependent excitations

$$
\begin{aligned}
& \frac{\mathrm{d} x^{\alpha}}{\mathrm{d} t}=\frac{c}{H_{A}^{+}}\left\{\frac{\left(1+\hat{\sigma}_{A} D^{2}\right)}{\left(1-\hat{\sigma}_{A} H^{2}\right)}\right. \\
& \left.\times\left(p^{\alpha}-(\boldsymbol{s} \cdot \boldsymbol{p}) s^{\alpha}\right)+(\boldsymbol{s} \cdot \boldsymbol{p}) s^{\alpha}\right\}, \\
& \frac{\mathrm{d} p_{\alpha}}{\mathrm{d} t} \propto p_{\alpha}, \quad \frac{\mathrm{d} p_{4}}{\mathrm{~d} t}=0 .
\end{aligned}
$$

Again, the case that $\boldsymbol{p}$ is parallel to $s$ leads to $v_{\mathrm{P}}=c$, so this case is of no interest for us. If, however, $\boldsymbol{p} \cdot \boldsymbol{s}=0$, 
we get

$$
\begin{aligned}
& H_{A}^{+}=c|\boldsymbol{p}| \sqrt{\frac{1+\hat{\sigma}_{A} D^{2}}{1-\hat{\sigma}_{A} H^{2}}}, \\
& v_{\mathrm{P}}=c \sqrt{\frac{1+\hat{\sigma}_{A} D^{2}}{1-\hat{\sigma}_{A} H^{2}}}, \\
& \frac{\mathrm{d} x^{\alpha}}{\mathrm{d} t}=c \sqrt{\frac{1+\hat{\sigma}_{A} D^{2}}{1-\hat{\sigma}_{A} H^{2}}} \frac{p^{\alpha}}{|\boldsymbol{p}|}
\end{aligned}
$$

and there is no deviation of a light ray from a straight line.

\section{AN INTERFEROMETRIC EXPERIMENT FOR TESTING NONLINEAR ELECTRODYNAMICS}

There are two ways in which Michelson interferometry can be used for testing nonlinear electrodynamics. First, a strong background field could be applied to the light beam in one arm of the interferometer. One would compare the situation where the background field is switched on with the situation where it is switched off, cf. [14]. Second, one could place the whole interferometer in a strong background field. One would then search for changes in the interference pattern if the interferometer is being rotated. The first possibility is reasonable if one thinks of a large interferometer, with an arm length of several meters at least. The second possibility is reasonable if one thinks of a tabletop interferometer. As an alternative to using a traditional Michelson interferometer, one could also use a pair of optical resonators as they have been used for high-precision Michelson-Morley experiments in recent years. As these resonators have a typical size of only a few centimeters, one would do the experiment with the whole instrument placed in a background field. With the resonators oriented perpendicularly to each other, one would then compare the situation where the field is switched on with the situation where it is switched off, or one would rotate the whole instrument with keeping the field switched on.

In the following we first discuss the setup of the experiment where a traditional Michelson interferometer is used and the field is placed in one arm. This is the variant which brings out the basic idea of the experiment most clearly. Later in this section we discuss the other variants.

Figure 1 shows the interferometer with the background field in the region denoted $B F$. The ray leaves the source $S$ and is divided at the semipermeable mirror $S P M$. After reflection at the mirrors $M_{1}$ and $M_{2}$, respectively, both parts interfere at $D$. If the background field is switched off, both parts always travel with the standard vacuum phase velocity $c$. If the background field is switched on, the part which travels along $l_{2}$ crosses

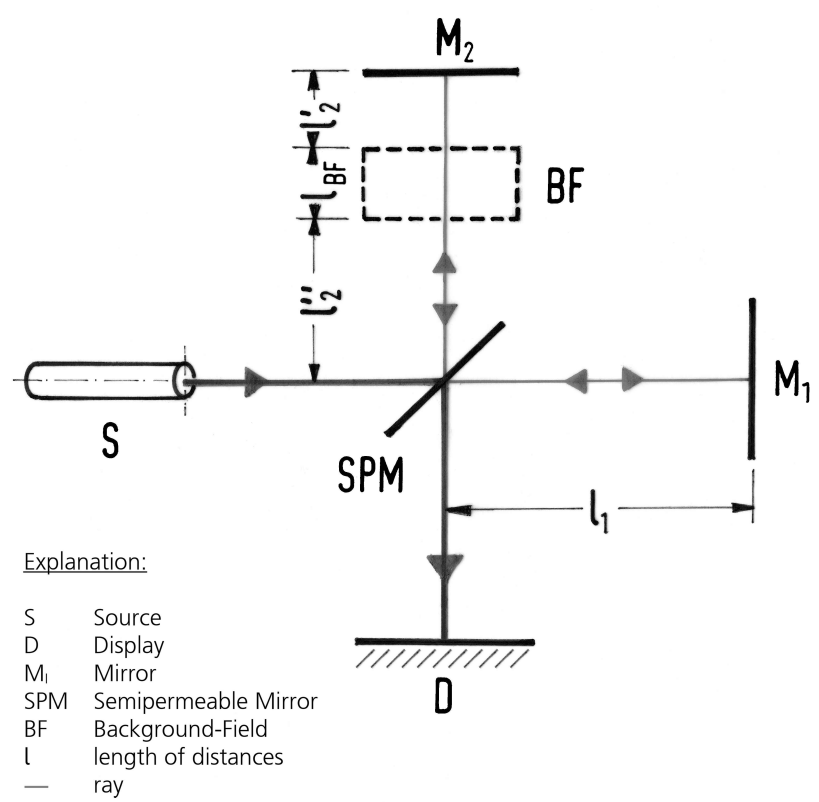

FIG. 1. Experimental setup

the region $B F$ with a different phase velocity, according to nonlinear electrodynamics. This would lead to a change of the interference pattern.

We consider the background field to be static, with one of the four fields $\boldsymbol{E}, \boldsymbol{B}, \boldsymbol{D}$, or $\boldsymbol{H}$ vanishing. Each of these four cases is covered by the calculations of the preceding section. We assume that the background field is perpendicular to the propagation direction of the light. We have seen that in this situation the ray does not deviate from a straight line.

Obviously the travel times of the ray along the different sections are given by

$$
c t_{1}=l_{1}, \quad c t_{2}^{\prime}=l_{2}^{\prime}, \quad c t_{2}^{\prime \prime}=l_{2}^{\prime \prime}, \quad v_{\mathrm{P}} t_{B F}=l_{B F} .
$$

Without background field the phase velocity is equal to $c$ everywhere, including the region $B F$. The time delay $\Delta t_{I}$ of the two arms is therefore given by

$$
\Delta t_{I}=2\left(t_{1}-t_{2}^{\prime}-t_{2}^{\prime \prime}-t_{B F}\right)=\frac{2}{c}\left(l_{1}-l_{2}^{\prime}-l_{2}^{\prime \prime}-l_{B F}\right) .
$$

With background field the phase velocity in the region $B F$ is $v_{\mathrm{P}}$ which is, in general, different from $c$. The time delay $\Delta t_{I I}$ of the two arms is therefore given by

$$
\begin{aligned}
\Delta t_{I I} & =2\left(t_{1}-t_{2}^{\prime}-t_{2}^{\prime \prime}-t_{B F}\right) \\
& =\frac{2}{c}\left(l_{1}-l_{2}^{\prime}-l_{2}^{\prime \prime}-\frac{c}{v_{\mathrm{P}}} l_{B F}\right) .
\end{aligned}
$$

The change of the interference pattern is given by the time difference

$$
\Delta t=\Delta t_{I I}-\Delta t_{I}=\frac{2 l_{B F}}{c}\left(1-\frac{c}{v_{\mathrm{P}}}\right) .
$$


This leads to a line shift of

$$
\Delta=\frac{\omega \Delta t}{2 \pi}=\frac{\omega l_{B F}}{\pi c}\left(1-\frac{c}{v_{\mathrm{P}}}\right) .
$$

Here $\omega$ denotes the frequency of the light. Note that $\omega$ is a constant because the background field is assumed static.

We evaluate the general result for each of the four cases $\boldsymbol{E}=\mathbf{0}, \boldsymbol{B}=\mathbf{0}, \boldsymbol{D}=\mathbf{0}$ and $\boldsymbol{H}=\mathbf{0}$. Note that in general $\boldsymbol{E}=\mathbf{0}$ is not equivalent to $\boldsymbol{D}=\mathbf{0}$ and $\boldsymbol{B}=\mathbf{0}$ is not equivalent to $\boldsymbol{H}=\mathbf{0}$.

a) Magnetostatic field strength $(E=0)$ :

From 52 we find that

$$
\begin{aligned}
v_{\mathrm{P}} & =c \sqrt{1+\sigma_{1 / 2} B^{2}} \\
& =c\left(1+\sigma_{1 / 2}(0) \frac{B^{2}}{2}+\ldots\right)
\end{aligned}
$$

and hence, by 64),

$$
\begin{aligned}
\Delta & =\frac{\omega l_{B F}}{\pi c}\left(1-\frac{1}{\sqrt{1+\sigma_{1 / 2} B^{2}}}\right) \\
& =\frac{\omega l_{B F} \sigma_{1 / 2}(0) B^{2}}{2 \pi c}+\ldots
\end{aligned}
$$

b) Electrostatic field strength $(B=0)$ :

From 52 we find that

$$
\begin{aligned}
v_{\mathrm{P}} & =c \frac{1}{\sqrt{1-\sigma_{1 / 2} E^{2}}} \\
& =c\left(1+\sigma_{1 / 2}(0) \frac{E^{2}}{2}+\ldots\right)
\end{aligned}
$$

and hence, by 64,

$$
\begin{aligned}
\Delta & =\frac{\omega l_{B F}}{c \pi}\left(1-\sqrt{1-\sigma_{1 / 2} E^{2}}\right) \\
& =\frac{\omega l_{B F} \sigma_{1 / 2}(0) E^{2}}{2 \pi c}+\ldots
\end{aligned}
$$

c) Magnetostatic excitation $(D=0)$ :

From (58) we find that

$$
\begin{aligned}
v_{\mathrm{P}} & =c \frac{1}{\sqrt{1-\hat{\sigma}_{1 / 2} H^{2}}} \\
& =c\left(1+\hat{\sigma}_{1 / 2}(0) \frac{H^{2}}{2}+\ldots\right)
\end{aligned}
$$

and hence, by 64),

$$
\begin{aligned}
\Delta & =\frac{\omega l_{B F}}{c \pi}\left(1-\sqrt{1-\hat{\sigma}_{1 / 2} H^{2}}\right) \\
& =\frac{\omega l_{B F} \hat{\sigma}_{1 / 2}(0) H^{2}}{2 \pi c}+\ldots
\end{aligned}
$$

\section{d) Electrostatic excitation $(H=0)$ :}

From (58) we find that

$$
\begin{aligned}
v_{\mathrm{P}} & =c \sqrt{1+\hat{\sigma}_{1 / 2} D^{2}} \\
& =c\left(1+\hat{\sigma}_{1 / 2}(0) \frac{D^{2}}{2}+\ldots\right)
\end{aligned}
$$

and hence, by (64),

$$
\begin{aligned}
\Delta & =\frac{\omega l_{B F}}{c \pi}\left(1-\frac{1}{\sqrt{1+\hat{\sigma}_{1 / 2} D^{2}}}\right) \\
& =\frac{\omega l_{B F} \hat{\sigma}_{1 / 2}(0) D^{2}}{2 \pi c}+\ldots
\end{aligned}
$$

If one writes $X$ for $E, B, D$, or $H$, one can combine all results up to first order in the form

$$
v_{\mathrm{P}}=c\left(1+\stackrel{X}{\sigma}_{1 / 2}(0) \frac{X^{2}}{2}+\ldots\right)
$$

and

$$
\Delta=\frac{\omega l_{B F} \stackrel{X}{\sigma_{1 / 2}}(0) X^{2}}{2 \pi c}+\ldots
$$

Here $\stackrel{X}{\sigma}_{1 / 2}(0)$ denotes either $\sigma_{1 / 2}(0)$ or $\hat{\sigma}_{1 / 2}(0)$, depending on whether $X$ is a field strength or an excitation. Note that $2 \pi c / \omega$ is the wavelength in the case of a vanishing background field. According to nonlinear electrodynamics, the wavelength changes when the ray travels through the background field $B F$. This means that, if one substitutes the angular frequency $\omega$ by the wavelength $\lambda=2 \pi c / \omega$,

$$
\Delta=\frac{l_{B F} \stackrel{X}{\sigma_{1 / 2}}(0) X^{2}}{\lambda}+\ldots
$$

one has to keep in mind that $\lambda$ is not the wavelength of the light when passing through the background field but of the light when emitted by the source.

The results of this section can also be applied to the case where the whole interferometer is inside the background field. Here one does not switch on and off the background field but rotates the interferometer by $90^{\circ}$ so that in the initial position the first arm is orthogonal to the field and the second arm is parallel to the field while in the end position it is vice versa. Then one gets instead of the preceding formulas the following ones:

$$
\begin{gathered}
\Delta t=\frac{2\left(l_{1}+l_{2}\right)}{c}\left(1-\frac{c}{v_{p}}\right), \\
\Delta=\frac{\omega\left(l_{1}+l_{2}\right) \stackrel{X}{\sigma_{1 / 2}}(0) X^{2}}{2 \pi c}+\ldots
\end{gathered}
$$


This means that one has to replace $l_{B F}$ by $l_{1}+l_{2}$ in all formulas to go from the fist setup to the second one.

As an alternative to using a traditional Michelson interferometer with two arms, we will now discuss a setup with optical resonators as it has been used frequently in recent years for high-precision Michelson interferometry; see e.g. 29] and the references therein. Here one uses a laser which is stabilized to the eigenfrequency $\nu_{\text {eigen }}=N v_{\mathrm{P}} /(2 L)$ of an optical resonator, where $N$ is the mode number, $v_{\mathrm{P}}$ is the phase velocity of light and $L$ is the length of the resonator. The quality of a resonator is determined by its finesse $F$, typically $F=100000$. In a figurative way, a resonator may be viewed as equivalent to a traditional interferometer whose arm length is folded $F$ times.

If $v_{\mathrm{P}}$ and $L$ undergo a change, the eigenfrequency of the resonator and therefore the frequency of the stabilized laser changes as

$$
\frac{\delta \nu}{\nu}=\frac{\delta v_{\mathrm{P}}}{v_{\mathrm{P}}}-\frac{\delta L}{L} .
$$

If the resonator is put into a homogeneous and static $\boldsymbol{E}$, $\boldsymbol{B}, \boldsymbol{D}$, or $\boldsymbol{H}$ field, with its axis perpendicular to the field, the phase velocity of light changes according to

$$
\frac{\delta v_{\mathrm{P}}}{v_{\mathrm{P}}} \approx \stackrel{X}{\sigma}_{1 / 2}(0) \frac{X^{2}}{2}
$$

if we use the approximations of 73 . As a direct measurement of $\delta \nu$ is not possible, one superimposes to the first laser a second reference laser stabilized to the eigenfrequency $\nu_{\text {ref }}$ of a resonator with (ideally) the same physical characteristics as the first one. Then the difference of the frequencies $\Delta \nu:=\nu_{\text {eigen }}-\nu_{\text {ref }}$ appears as the carrier frequency of the resulting beat. If the second resonator is oriented parallel to the background field and thus not influenced by it, this means that $\Delta \nu=\delta \nu$.

For a theoretical discussion of the effect, we assume that $L$ is not changed if the background field is applied. (Of course, for a practical realization of the experiment one has to take into account that the material of the resonator is influenced, e.g., by magnetostriction, but we ignore this here.) Then

$$
\frac{\delta \nu}{\nu}=\frac{\delta v_{\mathrm{P}}}{v_{\mathrm{P}}} \approx \stackrel{X}{\sigma}_{1 / 2}(0) \frac{X^{2}}{2} .
$$

In [29], by averaging over many measurements it was possible to determine $\delta \nu / \nu$ with an accuracy of $10^{-17}$. If we assume that the same accuracy can be reached in the experiment proposed here, a measurable effect requires that $\mathrm{X}$ satisfies

$$
\frac{\delta v_{\mathrm{P}}}{v_{\mathrm{P}}} \approx \stackrel{X}{\sigma}_{1 / 2}(0) \frac{X^{2}}{2} \approx 10^{-17} .
$$

In the next section we discuss the perspectives of performing such an experiment as a test of particular theories of the Plebański class. We compare the setup with the background field placed in one arm of a big Michelson interferometer with the setup using optical resonators. In the following, we refer to the first one as the "large-scale experiment" and to the second one as the "small-scale experiment."

\section{APPLICATION TO SPECIAL THEORIES OF THE PLEBAŃSKI CLASS}

\section{A. Born-Infeld theory}

In the case of the Born-Infeld theory, Lagrangian and Hamiltonian are given by [2]

$$
\begin{gathered}
\mathcal{L}(F, G)=-b_{0}^{2} \sqrt{1+\frac{F}{b_{0}^{2}}-\frac{G^{2}}{b_{0}^{4}}}+b_{0}^{2}, \\
\mathcal{H}(R, S)=b_{0}^{2} \sqrt{1+\frac{R}{b_{0}^{2}}-\frac{S^{2}}{b_{0}^{4}}}-b_{0}^{2},
\end{gathered}
$$

where $b_{0}$ is a new constant of Nature with the dimension of a field strength. The constitutive law reads

$$
H^{a b}=\frac{F^{a b}-\frac{G}{b_{0}^{2}} \tilde{F}^{a b}}{\sqrt{1+\frac{F}{b_{0}^{2}}-\frac{G^{2}}{b_{0}^{4}}}}
$$

which can be solved for the field strength,

$$
F^{m n}=\frac{H^{m n}+\frac{S}{b_{0}^{2}} \tilde{H}^{m n}}{\sqrt{1+\frac{R}{b_{0}^{2}}-\frac{S^{2}}{b_{0}^{4}}}} .
$$

The invariants $R$ and $S$ are given in terms of $F$ and $G$ by

$$
\begin{gathered}
\frac{R}{b_{0}^{2}}=\frac{-\frac{F}{b_{0}^{2}}+4 \frac{G^{2}}{b_{0}^{4}}+\frac{F}{b_{0}^{2}} \frac{G^{2}}{b_{0}^{4}}}{1+\frac{F}{b_{0}^{2}}-\frac{G^{2}}{b_{0}^{4}}}, \\
S=-G,
\end{gathered}
$$

which implies

$$
\frac{1+\frac{F}{b_{0}^{2}}-\frac{G^{2}}{b_{0}^{4}}}{1+\frac{G^{2}}{b_{0}^{4}}}=\frac{1+\frac{S^{2}}{b_{0}^{4}}}{1+\frac{R}{b_{0}^{2}}-\frac{S^{2}}{b_{0}^{4}}} .
$$

This leads to

$$
\begin{aligned}
\mathcal{L}_{F F} & =-\frac{2 \mathcal{L}_{F}^{3} G}{b_{0}^{2}}, \quad \mathcal{L}_{F G}=\frac{4 \mathcal{L}_{F}^{3} G}{b_{0}^{4}}, \\
\mathcal{L}_{G G} & =-\frac{2 \mathcal{L}_{F}}{b_{0}^{2}}-\frac{8 \mathcal{L}_{F}^{3} G^{2}}{b_{0}^{2}}, \\
\mathcal{H}_{R R} & =-\frac{2 \mathcal{H}_{R}{ }^{3} S}{b_{0}^{2}}, \quad \mathcal{H}_{R S}=\frac{4 \mathcal{H}_{R}{ }^{3} S}{b_{0}^{4}}, \\
\mathcal{H}_{S S} & =-\frac{2 \mathcal{H}_{R}}{b_{0}^{2}}-\frac{8 \mathcal{H}_{R}{ }^{3} S^{2}}{b_{0}^{2}} .
\end{aligned}
$$


Therefore one gets for the functions $\sigma_{1 / 2}$ and $\hat{\sigma}_{1 / 2}$, which give the deviation from the standard Maxwell vacuum theory,

$$
\begin{aligned}
& \sigma_{1}=\sigma_{2}=-\frac{1}{b_{0}^{2}+F}=-\frac{1}{b_{0}^{2}}+\cdots \\
& \hat{\sigma}_{1}=\hat{\sigma}_{2}=-\frac{1}{b_{0}^{2}+R}=-\frac{1}{b_{0}^{2}}+\ldots
\end{aligned}
$$

It is worth noticing that $\sigma_{1}=\sigma_{2}$ holds not only in the Born-Infeld theory and in the standard vacuum Maxwell theory but also in any other theory whose Lagrangian differs only by a term linear in G from them. (Such theories, however, are often excluded because they are not invariant under parity transformations.)

Additionally one can calculate the phase velocity and the line shift for the four static cases:

\section{Cases a) with $\mathrm{E}=\mathbf{0}$ and d) with $\mathrm{H}=\mathbf{0}$ :}

Here we discuss the case of a magnetostatic field strength and the case of an electrostatic excitation together. If we use the abbreviation $Y=B, D$, we find

$$
\stackrel{Y}{\sigma_{1}}=\stackrel{Y}{\sigma}_{2}=\frac{-1}{b_{0}^{2}+Y^{2}}
$$

hence

$$
v_{\mathrm{S}}=v_{\mathrm{P}}=\frac{c}{\sqrt{1+\frac{Y^{2}}{b_{0}^{2}}}}=c\left(1-\frac{Y^{2}}{2 b_{0}^{2}}+\cdots\right) .
$$

The limit $Y \rightarrow 0$ yields $v_{\mathrm{S}}=v_{\mathrm{P}} \rightarrow c$ as it has to.

By contrast, the limit $Y \rightarrow \infty$ yields $v_{\mathrm{S}}=v_{\mathrm{P}} \rightarrow 0$, so one may say that the background field $Y$ slows down the light ray. There is no upper bound for $Y=B, D$.

The line shift is given by

$$
\begin{aligned}
\Delta & =\frac{2 l_{B F}}{\lambda}\left(1-\sqrt{1+\frac{Y^{2}}{b_{0}^{2}}}\right) \\
& =-\frac{l_{B F}}{\lambda} \frac{Y^{2}}{b_{0}^{2}}+\ldots
\end{aligned}
$$

\section{Cases b) with $\mathrm{B}=\mathbf{0}$ and $\mathrm{c}$ ) with $\mathrm{D}=\mathbf{0}$ :}

Here we discuss the case of an electrostatic field strength and the case of a magnetostatic excitation together. If we use the abbreviation $Z=E, H$, we find

$$
\stackrel{Z}{\sigma_{1}}=\stackrel{Z}{\sigma_{2}}=\frac{1}{Z^{2}-b_{0}^{2}}
$$

hence

$$
v_{\mathrm{S}}=v_{\mathrm{P}}=c \sqrt{1-\frac{Z^{2}}{b_{0}^{2}}}=c\left(1-\frac{Z^{2}}{2 b_{0}^{2}}+\cdots\right) .
$$

Again, the limit $Z \rightarrow 0$ yields $v_{\mathrm{S}}=v_{\mathrm{P}} \rightarrow c$.

In contrast to the case above this one leads to an upper bound for $Z$. This is obvious because for $Z \rightarrow b_{0}$ one gets $v_{\mathrm{S}}=v_{\mathrm{P}} \rightarrow 0$. In analogy to the other cases a background excitation slows down the light ray. For background fields $Z>b_{0}$ one gets an imaginary phase velocity, so one has to conclude that $Z \leq b_{0}$.

The line shift is given by

$$
\begin{aligned}
\Delta & =\frac{2 l_{B F}}{\lambda}\left(1-\frac{1}{\sqrt{1-\frac{Z^{2}}{b_{0}^{2}}}}\right) \\
& =-\frac{l_{B F}}{\lambda} \frac{Z^{2}}{b_{0}^{2}}+\ldots
\end{aligned}
$$

We may combine the first-order approximations of all preceding cases into two formulas, one for the velocity and one for the line shift:

$$
v_{\mathrm{S}}=v_{\mathrm{P}} \approx c\left(1-\frac{X^{2}}{2 b_{0}^{2}}\right) \quad \text { and } \quad \Delta \approx-\frac{l_{B F}}{\lambda} \frac{X^{2}}{b_{0}^{2}} .
$$

To give an example we calculate the line shift for the large-scale experiment for some specific values. We assume an accuracy of about $10^{-6}$ line shifts. For $l_{B F}=$ $100 \mathrm{~m}$ and $\lambda=1000 \mathrm{~nm}$ one gets:

$$
\Delta=-\frac{l_{B F}}{\lambda} \frac{X^{2}}{b_{0}^{2}} \approx-10^{8} \frac{X^{2}}{b_{0}^{2}} .
$$

With these values one sees an effect if

$$
X \gtrsim 10^{-7} b_{0} .
$$

Born and Infeld conjectured that

$$
b_{0}=\frac{e}{r_{e}^{2}} \approx 6 \times 10^{15} \frac{\sqrt{\mathrm{g}}}{\sqrt{\mathrm{cm} \mathrm{s}}}
$$

where $e$ is the electron charge and $r_{e}$ is the classical electron radius. Although this is only of historical interest, we remark that the corresponding line shift would be

$$
\Delta \approx-\frac{10^{-22}}{36} X^{2} \frac{\mathrm{cm} \mathrm{s}^{2}}{\mathrm{~g}} .
$$

If this were true we would need a field strength or an excitation of

$$
X \gtrsim 6 \times 10^{8} \frac{\sqrt{\mathrm{g}}}{\sqrt{\mathrm{cm} \mathrm{s}}}
$$

to see an effect. For a magnetic field strength, $X=B$, this would correspond to $B_{\mathrm{SI}} \gtrsim 6 \times 10^{4} T$ in SI units, see (1). Clearly, this is not achievable in the foreseeable future.

It is more interesting to see what lower bound on $b_{0}$ one could get from an experiment. Let us assume that

$$
X \approx \times 10^{4} \frac{\sqrt{\mathrm{g}}}{\sqrt{\mathrm{cm} \mathrm{s}}}
$$


which corresponds to $B_{\mathrm{SI}}=1 \mathrm{~T}$ according to (1). This is not an unrealistic value for a magnetic field to be produced in a laboratory. Then a null result of our largescale experiment would imply, according to 100 , that

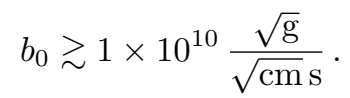

For the small-scale experiment we suggest a lower field strength of $300 \mathrm{mT}$ to prevent magnetorestriction. Then we find from 81 and $(91)$ that

$$
b_{0} \gtrsim 7 \times 10^{11} \frac{\sqrt{\mathrm{g}}}{\sqrt{\mathrm{cm} \mathrm{s}}}
$$

which is almost 2 orders of magnitude better than the large-scale experiment.

\section{B. Born's theory}

Born's Langrangian [1] differs from the Born-Infeld Lagrangian by omitting the $G^{2}$ term,

$$
\mathcal{L}(F)=-b_{0}^{2} \sqrt{1+\frac{F}{b_{0}^{2}}}+b_{0}^{2} .
$$

This leads to an electrodynamical theory with birefringence,

$$
\sigma_{1}=0, \quad \sigma_{2}=\frac{-1}{b_{0}^{2}+F} .
$$

From the viewpoint of geometrical optics Born's theory is a hybrid. One polarization mode behaves according to the standard vacuum Maxwell theory and the other one according to the Born-Infeld theory. This means that, if one filters the $\sigma_{2}$ rays out with a polarization filter, then one sees no difference to Maxwell, and if one filters the $\sigma_{1}$ rays out, then one sees no difference to Born-Infeld. As a consequence, the results of Sec. IV A are also valid for the $\sigma_{2}$ rays in Born's theory.

\section{Series expansions for electrodynamics with arbitrary Lagrangian}

If we are interested only in first-order deviations from Maxwell's theory, we may express the Langrangian in terms of a series expansion with respect to $F / A$ and $G / A$ up to second order, where $A$ is a constant with the dimension of a field strength squared. Introducing $A$ is necessary because only for dimensionless terms is it meaningful to say that they are small without referring to a particular system of units. In the Born-Infeld theory, e.g., we choose $A=b_{0}^{2}$.

The series expansion of the Lagrangian reads

$$
\begin{aligned}
\mathcal{L}=\alpha+ & \beta_{1} \frac{F}{A}+\beta_{2} \frac{G}{A}+ \\
& +\underset{1}{\gamma}\left(\frac{F}{A}\right)^{2}+\underset{2}{\gamma} \frac{F G}{A^{2}}+\gamma_{3}^{\gamma}\left(\frac{G}{A}\right)^{2}+\ldots
\end{aligned}
$$

Note that $\beta$ and $\gamma$ are zero if the theory is invariant under parity transformations. One can assume the validity of the following bookkeeping system for the smallness of terms, where $\sim$ means that terms are of the same order.

- $\alpha \sim \beta_{i} \sim \underset{i}{\gamma} \cdots$ as well as $F^{m n} \sim \tilde{F}^{m n}$, hence $F \sim$ G.

- $F / A$ and $G / A$ are dimensionless with $F / A \sim G / A$.

- From the first order of $R=R(F, G)$ and $S=$ $S(F, G)[\mathrm{cf.} 139$ to 145$]$ one gets $\underset{i}{\beta F / A \sim}$ $A R / \beta$.

With the help of $(139)$ to $(145)$ ) one can now calculate $R$ and $S$ as series in $F$ and $G$. Additionally one can then calculate the inverted series, i.e. $F$ and $G$ as a series in $R$ and $S$. This step allows us then to calculate the Hamiltonian as a function of $R$ and $S$. The result of this calculation is

$$
\begin{aligned}
\mathcal{H}=-\alpha & +\underset{1}{B} \frac{R}{\hat{A}}+\underset{2}{B} \frac{S}{\hat{A}}+ \\
& +\underset{1}{C}\left(\frac{R}{\hat{A}}\right)^{2}+\underset{2}{C} \frac{R S}{\hat{A}^{2}}+\underset{3}{C}\left(\frac{S}{\hat{A}}\right)^{2}+\cdots
\end{aligned}
$$

with the following coefficients:

$$
\begin{aligned}
& \hat{A}^{-1}:=\frac{A}{\beta_{2}^{2}+4 \beta^{2}} ; \quad B:=-\underset{1}{\beta} ; \quad \underset{2}{B}:=\beta_{2} ;
\end{aligned}
$$

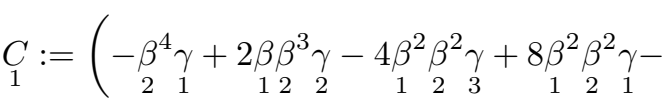

$$
\begin{aligned}
& \left.\begin{array}{rrr}
-8 \beta^{3} \beta \gamma-16 \beta^{4} \gamma \\
122 & 1 & 1
\end{array}\right) /\left(\begin{array}{c}
\beta^{2}+4 \beta^{2} \\
2
\end{array}\right)^{2} \\
& \underset{2}{C}:=\left(\begin{array}{rrrrr}
-\beta^{4} \gamma+4 \beta \beta^{3} \gamma-16 \beta \beta^{3} \gamma+24 \beta^{2} \beta^{2} \gamma- \\
2 & 12 & 3 & 12 & 1 \\
2 & & 1 & 2 & 2
\end{array}\right.
\end{aligned}
$$

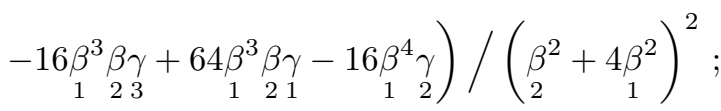

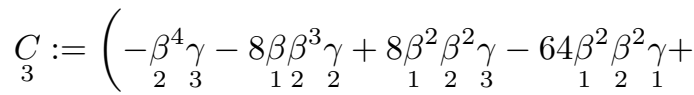

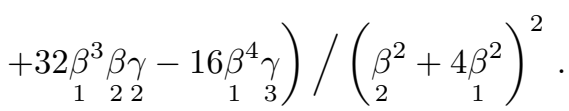

This leads to some additions to the bookkeeping system:

- From $\beta F / A \sim A R / \beta$ one gets $F / A \sim R / \hat{A}$ as well as $G / \hat{A} \sim S / \hat{A}$

- It is easy to see that $\alpha \sim \underset{i}{\beta} \sim \underset{i}{\gamma} \cdots \sim \underset{i}{B} \sim \underset{i}{C} \cdots$ holds.

As a result of this bookkeeping system one sees that, if one can neglect in the Lagrangian terms of a certain order 
in $F / A$ and $G / A$, then one can neglect in the Hamiltonian terms of the same order in $R / \hat{A}$ and $S / \hat{A}$.

For the case of a parity-invariant Lagrangian, $\mathcal{L}(F, G)=\mathcal{L}(F,-G)$, the coefficients of the Hamiltonian become very simple:

$$
\begin{aligned}
& \underset{1}{B}=-\underset{1}{\beta} ; \quad \underset{2}{B}=-\underset{2}{\beta}=0 ; \\
& \underset{1}{C}=-\underset{1}{\gamma} ; \quad \underset{2}{C}=-\underset{2}{\gamma}=0 ; \quad \underset{3}{C}=-\underset{3}{\gamma}, \\
& 4 \beta^{2} \\
& \hat{A}=\frac{1}{A} \text {. }
\end{aligned}
$$

If, in addition, the first-order approximation of the Lagrangian coincides with the standard Maxwell one (8) which is true if $\beta=-A / 2$ - one gets

$$
\hat{A}=A
$$

This is, in particular, the case for the theories of Born, Born-Infeld, and Heisenberg-Euler.

From the Lagrangian (or the Hamiltonian, respectively) one gets the "deviation coefficients" $\sigma_{1 / 2}$ in the zeroth order of approximation with respect to $F$ and $G$ (or $R$ and $S$, respectively):

$$
\begin{aligned}
& \sigma_{1 / 2}(0)=\frac{2 \gamma}{A \beta}+\frac{\gamma}{2 A \beta}
\end{aligned}
$$

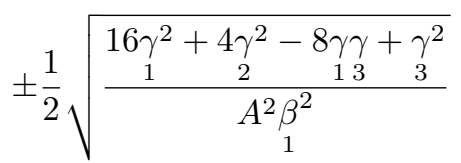

$$
\begin{aligned}
& \hat{\sigma}_{1 / 2}(0)=\frac{2 C_{1}}{\hat{A} B}+\frac{{ }_{1}^{C}}{2 \hat{A} B} \\
& \pm \frac{1}{2} \sqrt{\frac{16 C^{2}+4 C_{2}^{2}-8 C C_{3}+C_{3}^{2}}{\hat{A}^{2} B_{1}^{2}}} .
\end{aligned}
$$

Obviously the zeroth order approximation of $\sigma_{1 / 2}$ gives the first-order approximation of the optical metric for the deviation from Maxwell's theory and therefore also of the phase velocity.

The Born-Infeld theory, e.g., yields $\sigma_{1}(0)=\sigma_{2}(0)=$ $\hat{\sigma}_{1}(0)=\hat{\sigma}_{2}(0)=-1 / b_{0}^{2}$, so one recovers the values calculated above. Additionally one sees that the approximation procedure does not destroy the absence of birefrigence in the given order of approximation.

One gets the results for the four cases described in Sec. III if one feeds (115) and (114) into $(73)$ and $(74)$ :
Magnetostatic field strength case $(E=0)$ :

$$
\begin{aligned}
& v_{\mathrm{P}}=c+c \frac{B^{2}}{2}\left(\begin{array}{c}
2 \gamma \\
\frac{1}{A \beta} \\
1
\end{array}+\frac{3}{2 A \beta}\right.
\end{aligned}
$$

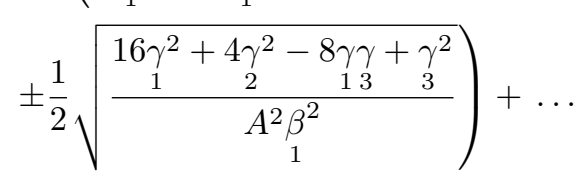

$$
\begin{aligned}
& \Delta=\frac{l_{B F} B^{2}}{\lambda}\left(\begin{array}{c}
2 \gamma \\
\frac{1}{A \beta} \\
1
\end{array}+\frac{3}{2 A \beta}\right.
\end{aligned}
$$

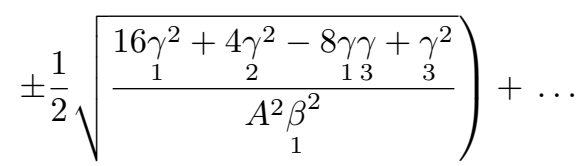

Electrostatic field strength case $(B=0)$ :

$$
\begin{aligned}
& v_{\mathrm{P}}=c+c \frac{E^{2}}{2}\left(\begin{array}{c}
2 \gamma \\
\frac{1}{A \beta} \\
1
\end{array}+\frac{3}{2 A \beta}\right.
\end{aligned}
$$

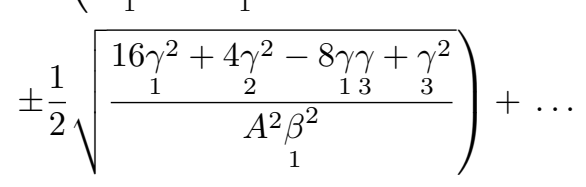

$$
\begin{aligned}
& \Delta=\frac{l_{B F} E^{2}}{\lambda}\left(\begin{array}{c}
2 \gamma \\
\frac{1}{A \beta} \\
1
\end{array}+\frac{3}{2 A \beta}\right.
\end{aligned}
$$

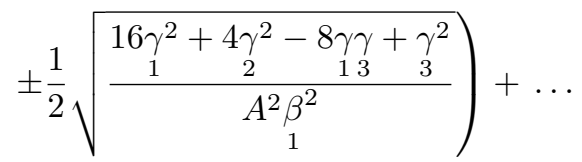

Magnetostatic excitation case $(D=0)$ :

$$
\begin{aligned}
v_{\mathrm{P}}=c & +c \frac{H^{2}}{2}\left(\frac{\underset{1}{2 C}+\frac{C_{3}}{\hat{A} B}+\frac{1}{2 \hat{A} B}}{1}\right. \\
& \left. \pm \frac{1}{2} \sqrt{\frac{16 C^{2}+4 C_{2}^{2}-8 C C_{3}+C_{3}^{2}}{\hat{A}^{2} B_{1}^{2}}}\right)+\ldots
\end{aligned}
$$

$$
\begin{array}{r}
\Delta=\frac{l_{B F} H^{2}}{\lambda}\left(\frac{2 C}{\hat{A} B}+\frac{3}{2 \hat{A} B}\right. \\
\left. \pm \frac{1}{2} \sqrt{\frac{16 C^{2}+\underset{1}{4 C_{2}^{2}-8 C C_{3}+C_{3}^{2}}}{\hat{A}^{2} B_{1}^{2}}}\right)+\ldots
\end{array}
$$


Electrostatic excitation case $(H=0)$ :

$$
\begin{aligned}
& v_{\mathrm{P}}=c+c \frac{D^{2}}{2}\left(\frac{2 C_{1}}{\hat{A} B}+\frac{C_{1}}{2 \hat{A} B}\right. \\
& \left. \pm \frac{1}{2} \sqrt{\frac{16 C^{2}+4 C_{2}^{2}-8 C_{3} C_{3}+C_{3}^{2}}{\hat{A}^{2} B_{1}^{2}}}\right)+\ldots \\
& \Delta=\frac{l_{B F} B^{2}}{\lambda}\left(\frac{\underset{1}{2 C}}{\hat{A} B}+\frac{3}{2 \hat{A} B}\right. \\
& \left. \pm \frac{1}{2} \sqrt{\frac{16 C^{2}+4 C_{2}^{2}-8 C_{3} C_{3}+C_{3}^{2}}{\hat{A}^{2} B^{2}}}\right)+\ldots
\end{aligned}
$$

In principle it is easy to obtain further orders of approximation, but the resulting terms are expected to be very small and will not be worked out here.

\section{The Heisenberg-Euler theory}

Here we give an example for the procedure described in the preceding section. For small values of the field strength the Heisenberg-Euler theory can be described by the following Lagrangian [3, 30, which results from a series expansion with respect to $F$ and $G$ :

$$
\mathcal{L}=E_{0}^{2}\left\{-\frac{1}{2} \frac{F}{E_{0}^{2}}+\Lambda\left(\frac{F^{2}}{E_{0}^{4}}+7 \frac{G^{2}}{E_{0}^{4}}\right)\right\}
$$

where

$$
\begin{gathered}
\Lambda=\frac{\hbar c}{90 \pi e^{2}}=0.7363 \\
E_{0}=\frac{m^{2} c^{4}}{e^{3}}=6.048 \times 10^{15} \frac{\sqrt{\mathrm{g}}}{\sqrt{\mathrm{cm} s}} .
\end{gathered}
$$

Here $e$ is the electron charge, $m$ is the electron mass, $c$ is the speed of light and $\hbar$ is Planck's constant.

So the coefficients in 109 are

$$
\begin{aligned}
& \alpha=0, \quad \underset{1}{\beta}=-\underset{1}{B}=-\frac{E_{0}^{2}}{2}, \quad \underset{2}{\beta}=0, \\
& \underset{1}{\gamma}=-\underset{1}{C}=\Lambda E_{0}^{2}, \quad \underset{2}{\gamma}=-\underset{2}{C}=0, \\
& \underset{3}{\gamma}=-\underset{3}{C}=7 \Lambda E_{0}^{2} .
\end{aligned}
$$

and

$$
\hat{A}=A=E_{0}^{2}
$$

Hence

$$
\begin{aligned}
& \sigma_{1}(0)=\hat{\sigma}_{1}(0)=-\frac{14 \Lambda}{E_{0}^{2}} \\
& \sigma_{2}(0)=\hat{\sigma}_{2}(0)=-\frac{8 \Lambda}{E_{0}^{2}}
\end{aligned}
$$

For the four possibilities for the background field described in Sec. III we get, using again the abbreviation $X=E, D, B, H$,

$$
\begin{aligned}
& v_{\mathrm{P}}\left(\sigma_{1}\right)=c\left(1-\frac{7 \Lambda X^{2}}{E_{0}^{2}}\right)+\cdots \\
& v_{\mathrm{P}}\left(\sigma_{2}\right)=c\left(1-\frac{4 \Lambda X^{2}}{E_{0}^{2}}\right)+\cdots \\
& \Delta\left(\sigma_{1}\right)=-\frac{14 \Lambda l_{B F} X^{2}}{\lambda E_{0}^{2}}+\cdots \\
& \Delta\left(\sigma_{2}\right)=-\frac{8 \Lambda l_{B F} X^{2}}{\lambda E_{0}^{2}}+\ldots
\end{aligned}
$$

Using the same setup as before for the large-scale experiment, with $l_{B F}=100 \mathrm{~m}$ and $\lambda=1000 \mathrm{~nm}$ one gets

$$
\begin{aligned}
& \Delta\left(\sigma_{1}\right) \approx-2 \times 10^{-23} X^{2} \frac{\mathrm{cm} \mathrm{s}^{2}}{\mathrm{~g}}, \\
& \Delta\left(\sigma_{2}\right) \approx-1 \times 10^{-23} X^{2} \frac{\mathrm{cm} \mathrm{s}^{2}}{\mathrm{~g}} .
\end{aligned}
$$

Therefore one needs a field strength or an excitation of

$$
X \gtrsim 3 \times 10^{8} \frac{\sqrt{\mathrm{g}}}{\sqrt{\mathrm{cm} s}}
$$

to see any effect. This is clearly not achievable with present or near-future instruments.

A similar calculation shows that for the small-scale experiment a field about 2 orders of magnitude smaller would be sufficient. However, even in this case one would need a field of more than $10^{6} \frac{\sqrt{\mathrm{g}}}{\sqrt{\mathrm{cm} \mathrm{s}}} \hat{=} 100 \mathrm{~T}$ to see an effect.

\section{CONCLUSIONS AND DISCUSSION}

Since Born and Infeld created their "new field theory" of electromagnetism 2], different nonlinear modifications of vacuum electrodynamics on the basis of a Lagrangian $\mathcal{L}(F, G)$ have been discussed, where usually one considers only those theories that reproduce the standard vacuum Maxwell theory in sufficiently weak fields. All these new electrodynamical theories have in common that they predict that light travels along the null cones of two optical metrics, one for each polarization state, where at least one of them differs from the vacuum Maxwell light-cone. At the same time they introduce at least one new dimensionfull constant of Nature.

While in the standard vacuum Maxwell theory the superposition principle holds, this is no longer true in other $\mathcal{L}(F, G)$ theories. As a consequence, an electromagnetic background field would have an effect on the propagation of electromagnetic waves and thus, in particular, on the phase velocity of light. This is reflected by the fact that the optical metrics depend on the background field. The best technique for measuring small changes in the phase velocity of light with high accuracy is interferometry. In 
this paper we worked out the mathematical details for using interferometry as a test of $\mathcal{L}(F, G)$ theories.

In cases where the constants of Nature that enter into the theory are known, as e.g. in the Heisenberg-Euler theory, an interferometric experiment could be used for confirming the theory by verifying the prediction. If instead the constants of Nature that enter into the theory are not known, as e.g. in the Born-Infeld theory, a null result of the experiment would give bounds on these constants. Our estimates demonstrate that, with realistic (magnetic) fields, an interferometric experiment could place significant bounds on the Born-Infeld constant $b_{0}$.

Unfortunately, in the case of the Heisenberg-Euler theory our estimates seem to indicate that a confirmation of the theory is not realizable with electromagnetic fields that can be achieved in present-day experiments. However, it might be possible to considerably enhance the sensitivity by using time-dependent background fields, rather than the static fields we have considered for our numerical estimates. For the case of testing the Heisenberg-Euler theory with an interferometer of the size of a gravitational wave detector, this possibility was discussed in detail recently by Grote [18. The idea is to change the background field periodically with a frequency $\omega$, e.g. by rotating a permanent magnet. As long as $\omega$ is small in comparison to the frequency of the laser light used in the interferometer, our equations could still be used for this situation in the sense of an adiabatic approximation. If the laser light is polarized, rotating the background field would lead to a periodically varying signal according to any theory that predicts birefringence in vacuo. (Unfortunately, this excludes the Born-Infeld theory.) By choosing long integration times - Grote suggests to run the experiment for a year one could improve the statistics in such a way that it might be possible to reach the sensitivity for testing the Heisenberg-Euler theory. A similar analysis has not been carried through for the small-scale experiment so far. We will leave this for other authors, as it goes beyond the scope of the present paper which was to lay the theoretical foundations of the experiment in the context of an arbitrary $\mathcal{L}(F, G)$ theory.

Finally, we add a remark on pulsed background fields. Pulsed magnetic fields and also laser pulses (pulsed null fields) can be produced with considerably higher field strengths than static or slowly varying fields. For example, pulsed magnetic fields of $\approx 100 \mathrm{~T}$ have already been produced in the laboratory. However, these fields persist only for short times, so the adiabatic approximation would not be valid which makes the theory considerably more difficult. Moreover, there are several technical obstacles. For example, we see major experimental difficulties towards a realization of the small-scale experiment with (pulsed) magnetic fields of $\approx 100 \mathrm{~T}$ because of magnetostriction. Also, for the experiment with a pulsed null field as a background one would wish to have the pulse traveling in the same direction as the laser beam in the interferometer, to make sure that the latter does not de- viate from a straight line. This cannot be done without changing the geometry of the interferometer, neither for the small-scale nor for the large-scale experiment. For these reasons, we have restricted our specific calculations to time-independent background fields (which includes the case of slowly varying fields in the sense of an adiabatic approximation).

\section{ACKNOWLEDGMENTS}

G.S. wishes to thank Evangelisches Studienwerk Villigst for supporting him with a Ph.D. stipend during the course of this work. V.P. is grateful to Deutsche Forschungsgemeinschaft for financial support under Grant No. LA 905/14-1. Moreover, we gratefully acknowledge support from the Deutsche Forschungsgemeinschaft within the Research Training Group 1620 "Models of Gravity." We also thank Sven Herrmann for helpful discussions on the experimental aspects of the subject and an anonymous referee for directing our attention to some important references.

\section{APPENDIX: HAMILTONIAN FORMALISM IN TERMS OF THE EXCITATION}

First we give a necessary and sufficient condition for the constitutive law (9) to be locally solvable for $F^{a b}$. By the implicit function theorem, this is true if the Jacobian of the map from the field strength 6 -vector to the excitation 6 -vector is nonzero After dividing by the factor $\left(4 \mathcal{L}_{F}^{2}+\mathcal{L}_{G}^{2}\right)^{2}$, which is nonzero unless the Lagrangian is constant and thus trivial, we find that this condition reads

$$
\begin{gathered}
4 \mathcal{L}_{F}^{2}+\mathcal{L}_{G}^{2}-4\left(F^{2}+4 G^{2}\right) \mathcal{L}_{F F} \mathcal{L}_{G G}+4\left(F^{2}+4 G^{2}\right) \mathcal{L}_{F G}^{2} \\
+8 F \mathcal{L}_{F} \mathcal{L}_{F F}+16 G \mathcal{L}_{F} \mathcal{L}_{F G}+4 F \mathcal{L}_{G G} \mathcal{L}_{G}-2 F \mathcal{L}_{F} \mathcal{L}_{G G} \\
-8 G \mathcal{L}_{F F} \mathcal{L}_{G}+2 G \mathcal{L}_{G} \mathcal{L}_{G G} \neq 0 .
\end{gathered}
$$

It is easy to see that this condition is satisfied, for all field configurations, in the Born theory and also in the Born-Infeld theory. For the Heisenberg-Euler Lagrangian (124) it is true as well, where we have to observe that this second-order theory is valid only as long as the magnitude of the field strength is small in comparison to $E_{0}$.

Whenever the constitutive law (5) can be solved for $F_{m n}$, we can pass to a Hamiltonian description by a Legendre transformation (12). In this appendix we derive some relevant equations of the Hamiltonian formalism that will be used in the body of the paper, based on an analogue formalism that was developed already by Born and Infeld [2] for their special theory.

From 12 and (5) we find

$$
\frac{\partial \mathcal{H}}{\partial H^{i j}}=-F_{i j}
$$


which is the Hamiltonian version of the constitutive law. In the case of vanishing sources, $j^{m}=0$, the Maxwell equations read

$$
\partial_{n} H^{m n}=0 \quad \text { and } \quad \partial_{[a} F_{b c]}=0 .
$$

These two equations can be equivalently rewritten as

$$
\partial_{[a} \tilde{H}_{b c]}=0 \quad \text { and } \quad \partial_{n} \tilde{F}^{m n}=0 .
$$

Comparison of (5) and (135) on one side and (134) and (136) on the other side demonstrates that the source-free theory is invariant under a duality rotation

$$
F^{m n} \hookrightarrow \tilde{H}^{m n}, \quad \mathcal{L} \hookrightarrow \mathcal{H} .
$$

In 3-vector notation, $F^{m n} \hookrightarrow \tilde{H}^{m n}$ means $E_{\alpha} \hookrightarrow H_{\alpha}$ and $B_{\alpha} \hookrightarrow-D_{\alpha}$. Clearly, $F^{m n} \hookrightarrow \tilde{H}^{m n}$ implies

$$
\tilde{F}^{m n} \hookrightarrow-H^{m n}, \quad F \hookrightarrow R, \quad G \hookrightarrow S .
$$

If we start from the Lagrangian $\mathcal{L}\left(F_{m n}\right)$ and work out all relevant equations of the theory in terms of the field strength, we get the relevant equations in terms of the excitation simply by applying the replacements (137) and (138). Note that this method works only in the case of vanishing sources, $j^{m}=0$, but for any Lagrangian $\mathcal{L}\left(F_{m n}\right)$ for which the constitutive law (5) can be solved for $F_{m n}$.

We now specify to a Lagrangian of the Plebański class. We recall that in this case the constitutive law reads

$$
H^{a b}=-2 \mathcal{L}_{F} F^{a b}+\mathcal{L}_{G} \tilde{F}^{a b} .
$$

Similarly, 134 specifies to

$$
F_{a b}=2 \mathcal{H}_{R} H_{a b}-\mathcal{H}_{S} \tilde{H}_{a b} .
$$

Inserting 139 into 12 yields

$$
\mathcal{H}(R, S)=2 \mathcal{L}_{F} F+2 \mathcal{L}_{G} G-\mathcal{L}(F, G)
$$

while inserting 140 into 12 yields

$$
\mathcal{H}(R, S)=2 \mathcal{H}_{R} R+2 \mathcal{H}_{S} S-\mathcal{L}(F, G) .
$$

From these two equations we read that

$$
\mathcal{L}_{F} F+\mathcal{L}_{G} G=\mathcal{H}_{R} R+\mathcal{H}_{S} S .
$$

Also, from 139 we find immediately that

$$
\begin{aligned}
& R=\left(-4 \mathcal{L}_{F}^{2}+\mathcal{L}_{G}^{2}\right)^{2} F-8 \mathcal{L}_{F} \mathcal{L}_{G} G, \\
& S=\left(-4 \mathcal{L}_{F}^{2}+\mathcal{L}_{G}^{2}\right)^{2} G+2 \mathcal{L}_{F} \mathcal{L}_{G} F .
\end{aligned}
$$

Similarly, from 140 we find that

$$
\begin{gathered}
F=\left(-4 \mathcal{H}_{R}^{2}+\mathcal{H}_{S}^{2}\right)^{2} R-8 \mathcal{H}_{R} \mathcal{H}_{S} S, \\
G=\left(-4 \mathcal{H}_{R}^{2}+\mathcal{H}_{S}^{2}\right)^{2} S+2 \mathcal{H}_{R} \mathcal{H}_{S} R .
\end{gathered}
$$

In Sec. IV C the equations (139) to 145 are used for calculating series expansions of the Lagrangian and the Hamiltonian theory up to second order in $F$ and $G$. This enables one to calculate the first post-Maxwellian results of the discussed experiment for an arbitrary Lagrangian of the Plebański class.
[1] M. Born, Nature 132, 282 (1933).

[2] M. Born and L. Infeld, Proc. R. Soc. London A 144, 425 (1934).

[3] W. Heisenberg and H. Euler, Zeitschrift für Physik 98(11), 714 (1936).

[4] J. Plebański, Lectures on non-linear electrodynamics (Niels Bohr Institute and NORDITA, Copenhagen, 1970).

[5] G. Boillat, J. Math. Phys. 11, 941 (1970).

[6] I. Białynicki-Birula, in Quantum Theory of Particles and Fields: Birtday volume dedicated to Jan Łopuszański, edited by B. Jancewicz and J. Lukierski (World Scientific, Singapore, 1983).

[7] A. A. Tseytlin, in The many faces of the superworld: Yuri Golfand memorial volume, edited by M. Shifman (World Scientific, Singapore, 1999).

[8] J. S. Toll, Ph.D. thesis, Princeton (1952).

[9] D. L. Burke, R. C. Field, G. Horton-Smith, J. E. Spencer, S. C. B. D. Walz, K. S. W. M. Bugg, A. W. Weidemann, C. Bula, K. T. McDonald, E. J. Prebys, et al., Phys. Rev. Lett. 79, 1626 (1997).

[10] D. d'Enterria and G. G. da Silveira, Phys. Rev. Lett. 111, 080405 (2013).

[11] F. D. Valle, U. Gastaldi, G. Messineo, E. Milotti,
R. Pengo, L. Piemontese, G. Ruoso, and G. Zavattini, New J. Phys. 15, 053026 (2013).

[12] R. Ferraro, Phys. Rev. Lett. 99, 230401 (2007).

[13] T. Dereli and R. Tucker, Europhys. Lett. 89, 20009 (2010).

[14] D. Boer and J.-W. van Holten, arXiv :hep-ph/0204207 (2002).

[15] V. I. Denisov, I. V. Krivchenkov, and N. V. Kravtsov, Phys. Rev. D. 69, 066008 (2004).

[16] B. Döbrich and H. Gies, Europhys. Lett. 87, 21002 (2009).

[17] G. Zavattini and E. Calloni, Eur. Phys. J. C 62, 459 (2009).

[18] H. Grote, Phys. Rev. D 91, 022002 (2015).

[19] J. D. Jackson, Classical Electrodynamcs, 3rd ed. (John Wiley \& Sons, Hoboken, 1999).

[20] F. W. Hehl and Y. N. Obukhov, Foundations of Classical Electrodynamics (Birkhäuser, Basel, 2003).

[21] Y. N. Obukhov and G. F. Rubilar, Phys. Rev. D 66, 024042 (2002).

[22] M. Novello, V. A. De Lorenci, J. M. Salim, and R. Klippert, Phys. Rev. D 61, 045001 (2000).

[23] M. Visser, C. Barceló, and S. Liberati, arXiv :grqc/0204017 (2008). 
[24] W. Dittrich and H. Gies, Phys. Rev. D. 58, 025004 (1998).

[25] G. M. Shore, Nucl. Phys. B 778, 219258 (2007).

[26] M. Kline and I. W. Kay, Electromagnetic Theory and Geometrical Optics (Interscience, New York, 1965).

[27] V. Perlick, J. Math. Phys. (N.Y.) 52, 042903 (2011).
[28] R. Courant and D. Hilbert, Methods of Mathematical Physics, Vol. II (Wiley Interscience Publishers, New York, 1962).

[29] S. Herrmann, A. Senger, K. Möhle, M. Nagel, E. V. Kovalchuk, and A. Peters, Phys. Rev. D. 80, 105011 (2009).

[30] G. Dunne, Int. J. Mod. Phys. A 27, 1260004 (2012). 